\title{
Nerve fibre velocity and refractory period distributions in nerve trunks
}

\author{
R. P. BETTS, D. M. JOHNSTON, AND B. H. BROWN \\ From the Area Medical Physics Department, and the Department of Child Health, \\ University of Sheffield, Sheffield
}

SYNOPSIS With the use of a double stimulus technique, nerve fibre velocity range measurements were performed over a single conduction distance in 13 normal subjects and over two conduction distances in another 12 normal subjects. The velocity ranges were found to be dependent upon the conduction distance, owing to unknown refractory period delays. Refractory period values were calculated for the 12 subjects and also direct refractory period distribution measurements were made on 15 normal subjects using a twin stimulus and automatic subtraction technique. Corrections to the velocity range measurements were made upon differing assumptions as to the correlation between refractory period and fibre conduction velocity. It was concluded that a single median value refractory period obtained from the distribution was the best correction to use, based upon the hypothesis that for group A fibres the random scatter of refractory period values is far greater than any variation due to a correlation between refractory period and fibre conduction velocity. It was found important to recognize that calculated values of velocity range are a function not only of the spread of fibre conduction velocities but also of refractory periods.

There is evidence of selective involvement of nerve fibres in some disorders where clinical involvement of the peripheral nerve is undetectable, such as motor neurone disease (Miglietta, 1968; Chaco, 1970) and mononeuritis (Hopf, 1963). It has been suggested that some of the so-called myopathic diseases contain a neuropathic element (McComas et al., 1970). Consequently, any means of defining more precisely nerve fibre abnormality will be valuable, and one possibility is the measurement of the distribution of fibre conduction velocities in a whole nerve trunk.

Many current measurement techniques allow only the fastest conduction velocities to be recorded since the more slowly conducting fibres have the onset of their action potentials hidden by the discharge of the faster units. A technique using two stimulating pulses has been proposed by several workers for the measurement of maximum and minimum conduction velocities within a nerve bundle (Thomas et al.,
1959; Hopf, 1963; Miglietta, 1966, 1968; Chaco, 1970). The method applies two supramaximal stimuli in sequence to a nerve. The first stimulus is applied close to, and the second at a greater distance from, the muscle supplied by the nerve. The muscle action potential resulting from the second stimulus varies in amplitude depending on the delay between the two stimuli owing to a collision cancellation effect of the afferent and efferent volleys. The time interval between the minimal and maximal amplitude responses, less any refractory period delays, is the difference in conduction delay between the fastest and slowest fibres. The conduction delay is dependent on the conduction rates of the nerve fibres and the delay due to the refractory period of the fibres.

We have carried out studies using the double pulse technique on normal subjects over varying lengths of the peripheral nerve in order to explain the discrepancies in velocity range values quoted by others (Hopf, 1963; Miglietta, 1968; Chaco, 1970). We have also devised a method of 
independently measuring the refractory period distribution within a nerve trunk in order to correct the conduction delay determined by the double pulse technique.

\section{METHODS}

VELOCITY RANGE A double pulse stimulator was used which delivered isolated rectangular electrical pulses of $100 \mu \mathrm{s}$ duration, with a pulse interval variable from 0 to $10 \mathrm{~ms}$. The repetition frequency was $1 \mathrm{~Hz}$. Surface electrodes used for stimulation and recording were strips of lead foil $3 \mathrm{~cm}$ by $0.5 \mathrm{~cm}$ placed $1 \mathrm{~cm}$ apart, and making contact with the skin through a conducting jelly. The muscle action potentials were amplified with a Tektronix 2A61 differential amplifier. In order to increase the accuracy of the observation of the maximal and minimal amplitude of the second response a graphical display unit was developed which plotted on a storage oscilloscope (Tektronix 564), the amplitude of the second response against the pulse interval delay. Figure 1 shows a Polaroid photograph of this type of graphical display.

Measurements were made on 25 normal subjects, whose ages ranged from 21 to 48 years (mean 26.3 years). The initial investigation was a measure of the maximal conduction velocity, using standard techniques, of the action potential evoked by a single supramaximal stimulus applied at two sites along a nerve, and recording the stimulus to response intervals from a muscle supplied by this nerve. The ulnar nerve was used in this study, the stimuli applied at the wrist and elbow, and the recording electrodes placed over the abductor digiti minimi muscle. The double pulse technique was then used, the first stimulus at the wrist, the second either at the elbow or, in the study of a longer nerve distance, on the niedial aspect of the upper arm. Throughout, the recordings were made under isometric conditions with the subject lying, in a warm room.

REFRACTORY PERIOD The recording equipment and stimulator were identical with those used for the double pulse velocity range method. The stimuli were applied at a single point over the ulnar or median nerve at the wrist. A monopolar electrode configuration was used for recording nerve action potentials from the nerve at the elbow. Paired supramaximal stimuli, with variable time intervals, were applied at this single point, and the two resulting nerve action potentials recorded. At short stimulus time intervals the two nerve action potentials begin to overlap and it is not possible to identify clearly the potential resulting from the second stimulus. This is illustrated in Fig. 2 which shows the two triphasic action potentials beginning to overlap. The resultant complex signal makes it impossible to measure all the latency and duration parameters of the second action potential. An automatic subtraction technique has been devised which overcomes the difficulty of isolating the second response. Initially, supramaximal twin stimuli were applied to the nerve and the recorded nerve action potentials passed through an eight bit analogue to digital converter and stored in a memory consisting of eight parallel 128 bit shift registers. Subsequently, a single supramaximal stimulus was applied and the resultant nerve action potential was fed to an analogue subtractor synchronously with the readout,

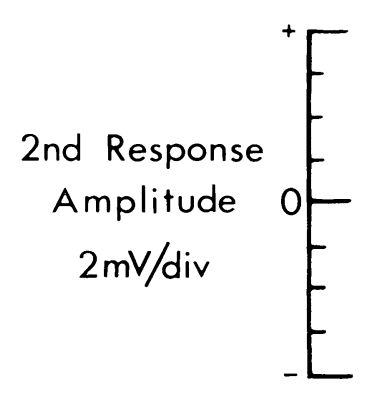

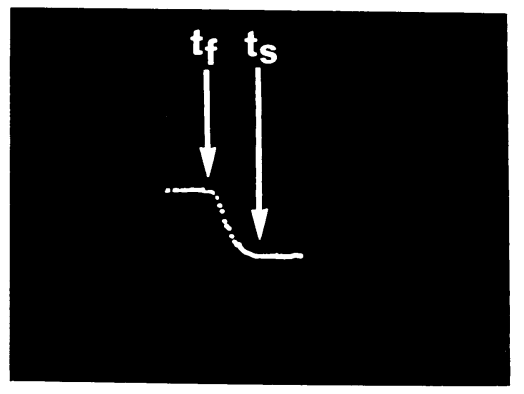

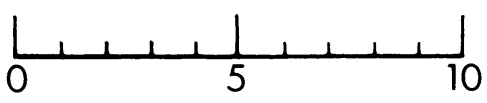

Pulse Interval (ms)
FIG. 1 A typical Polaroid photograph of the graphical display used in the velocity range test, showing the change in amplitude of the second muscle action potential with varying palse interval. 


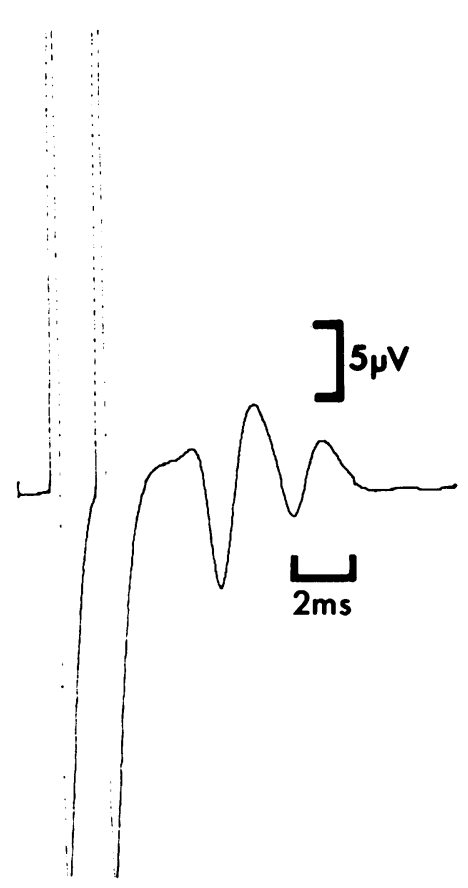

FIG. 2 Two nerve action potentials beginning to overlap at short time intervals between two stimuli.

via a digital to analogue converter, from the memory. Since the nerve action potential obtained from the single stimulus is the same as the nerve action potential obtained from the first of the twin stimuli, the output of the analogue subtractor is the response to the second of the twin stimuli only. By repeatedly applying double then single stimuli an averaged output of the subtractor was obtained.

The output of the subtractor unit was averaged on a Biomac 1010 computer and displayed on a Bryans 26000 A3 $X-Y$ plotter. The recording conditions were identical with those used for the velocity range measurements. A series of recordings was obtained with varying time intervals of the paired stimuli.

Measurements have been made on 15 normal subjects with an age range of 7 to 36 years (mean 20 years).

\section{RESULTS}

RANGE OF CONDUCTION VELOCITIES The results of the conduction velocity range measurements are given in Table 1. The maximum conduction velocities are within the range of $53.1-66.7 \mathrm{~m} / \mathrm{s}$ for 24 subjects, with a mean of $59.2 \pm 7.6 \mathrm{~m} / \mathrm{s}$ $( \pm 2 \mathrm{SD})$. The velocity range, the difference between the fastest and slowest rates, is given as $D / t_{f}-D / t_{s}$, where $D$ is the conduction distance, and $t_{f}$ and $t_{s}$ are the delay intervals to the minimal and maximal amplitude responses respectively, as shown in Fig. 1. Any effects imposed by refractory period delays have been ignored in this calculation. The velocity ranges over the short and long distances are given in Table 1.

TABLE 1

VELOCITY RANGE MEASUREMENTS

\begin{tabular}{|c|c|c|c|c|c|}
\hline \multirow[t]{3}{*}{ Subject } & \multirow{3}{*}{$\begin{array}{c}\text { Maximum } \\
\text { conduction } \\
\text { velocity } \\
(\mathrm{m} / \mathrm{s})\end{array}$} & \multicolumn{2}{|c|}{ Velocity range $(\mathrm{m} / \mathrm{s})$} & \multirow{2}{*}{\multicolumn{2}{|c|}{$\begin{array}{c}\text { Calculated refractory } \\
\text { periods } \\
(\mathrm{ms})\end{array}$}} \\
\hline & & \multirow{2}{*}{$\begin{array}{l}\text { Short } \\
\text { distance }\end{array}$} & \multirow{2}{*}{$\begin{array}{l}\text { Long } \\
\text { distance }\end{array}$} & & \\
\hline & & & & Minimum & Maximum \\
\hline 1 & 60.0 & 10.0 & & & \\
\hline 2 & 54.5 & 8.2 & & & \\
\hline 3 & 60.0 & 10.9 & & & \\
\hline 4 & 57.3 & 9.3 & & & \\
\hline 5 & 57.0 & 6.9 & & & \\
\hline 6 & 62.5 & 11.9 & & & \\
\hline 7 & 56.5 & 8.9 & & & \\
\hline 8 & 56.5 & 12.0 & & & \\
\hline 9 & - & 9.1 & 8.1 & 1.14 & 1.95 \\
\hline 10 & 55.8 & 9.4 & 9.0 & 1.35 & 2.33 \\
\hline 11 & 64.0 & 9.3 & 8.5 & 0.82 & 1.56 \\
\hline 12 & 53.8 & 9.3 & 8.0 & 1.40 & 3.00 \\
\hline 13 & 62.1 & 7.7 & & & \\
\hline 14 & 56.7 & 8.5 & & & \\
\hline 15 & 63.4 & 9.8 & 9.0 & 1.44 & 2.60 \\
\hline 16 & 62.8 & 8.8 & 8.5 & 1.62 & 2.60 \\
\hline 17 & 62.5 & 7.1 & & & \\
\hline 18 & 59.0 & 7.7 & 6.5 & 0.40 & 1.05 \\
\hline 19 & 56.5 & 8.7 & 7.9 & 1.40 & 2.30 \\
\hline 20 & 66.7 & 9.3 & & & \\
\hline 21 & 58.7 & 11.1 & & & \\
\hline 22 & 53.1 & 11.4 & 11.3 & 2.10 & 3.80 \\
\hline 23 & 58.8 & 10.1 & 9.7 & 1.78 & 3.07 \\
\hline 24 & 55.8 & 11.9 & 10.3 & 0.80 & 2.20 \\
\hline 25 & 65.8 & 10.4 & 9.7 & 1.70 & 3.00 \\
\hline
\end{tabular}

The refractory period values have been calculated by allowing an inverse relationship between refractory period and conduction velocity rates. Taking the latency measurements $t_{f}$ and $t_{s}$ for the long and short conduction distances, and knowing these distances, then these values may be fitted to a simple set of simultaneous equations which may be solved to give the theoretical refractory periods. The equations are simply of the form velocity equals distance divided by time where a refractory period correction value is included in the time 
parameter. As shown in Table 1 , the range of $0.8-2.1 \mathrm{~ms}$ is obtained for the fast fibres, and $1.05-3.8 \mathrm{~ms}$ for the slow fibres.

REFRACTORY PERIOD The results of the refractory period measurements are given in Table 2. In Fig. 3 are shown the $X-Y$ plotter

TABLE 2

DIRECT MEASUREMENTS OF REFRACTORY PERIOD

\begin{tabular}{|c|c|c|c|}
\hline \multirow[t]{2}{*}{ Subject } & \multicolumn{3}{|c|}{ Refractory period $(\mathrm{ms})$} \\
\hline & Minimum & Maximum & Median \\
\hline 1 & 0.775 & 3.3 & 1.50 \\
\hline 2 & 0.700 & 3.0 & 1.10 \\
\hline 3 & - & 2.8 & 1.45 \\
\hline 4 & 0.725 & 3.0 & 1.24 \\
\hline 5 & 0.650 & 2.0 & 1.00 \\
\hline 6 & 0.875 & 3.4 & 1.45 \\
\hline 7 & 0.850 & 3.1 & 1.50 \\
\hline 8 & 0.750 & - & - \\
\hline 9 & 0.675 & 3.0 & 1.03 \\
\hline 10 & 0.725 & 2.7 & 1.37 \\
\hline 11 & 0.700 & 3.5 & 0.94 \\
\hline 12 & 0.750 & 2.9 & 1.53 \\
\hline 13 & 0.700 & - & - \\
\hline 14 & 0.775 & 2.5 & 1.40 \\
\hline 15 & 0.750 & 3.6 & 1.40 \\
\hline
\end{tabular}

readouts of the nerve action potential resulting from the second stimulus. Figure 4 shows a graph of the amplitude of the second nerve action potential normalized to its maximum value, plotted against the delay between the two stimuli, which can be taken as a measure of the refractory periods. The maximum refractory period for 13 subjects was $3.06 \pm 0.66 \mathrm{~ms}$ ( $\pm 2 \mathrm{SD}$ ), the minimum refractory period for 14 subjects was $0.74 \pm 0.12 \mathrm{~ms}( \pm 2 \mathrm{SD})$. In three of the subjects the full range of values could not be obtained because of poor signal to noise levels. Figure 5 shows the differentiation of the curve in Fig. 4 normalized to the peak value. The median refractory period values calculated for this distribution are given in Table 2.

\section{DISCUSSION}

Normal ranges are given in the literature for the difference between fastest and slowest nerve conduction velocities but there are discrepancies between workers. Chaco (1970) and Miglietta

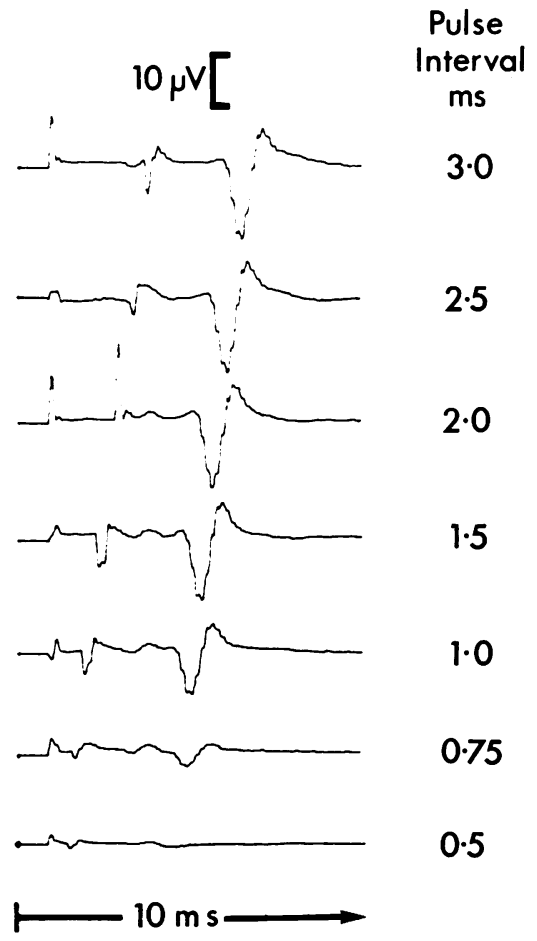

FIG. $3 A$ set of $X-Y$ plotter readouts from the refractory period test, showing the change of amplitude of the second nerve action potential with varying pulse interval.

(1968) applying the double pulse technique between wrist and elbow, Chaco examining the ulnar nerve and Miglietta the ulnar and median nerves, quote a normal range of velocities of $7-12 \mathrm{~m} / \mathrm{s}$. Hopf (1963), however, using the axilla to wrist length of the ulnar nerve, gives a range of $4-7 \mathrm{~m} / \mathrm{s}$. Miglietta (1966) gives a normal range of $4-7 \mathrm{~m} / \mathrm{s}$ for the deep peroneal nerve. These velocity ranges are much narrower than might be expected. The failure to take refractory period delays into account, which are significant percentages of the delay times measured, which in turn are a function of conduction distance, may explain the narrow range of velocities.

There are few measurements of the refractory periods of human peripheral nerves on which to base corrections of the conduction times determined by the double pulse technique. The pulse interval at which the nerve failed to con- 


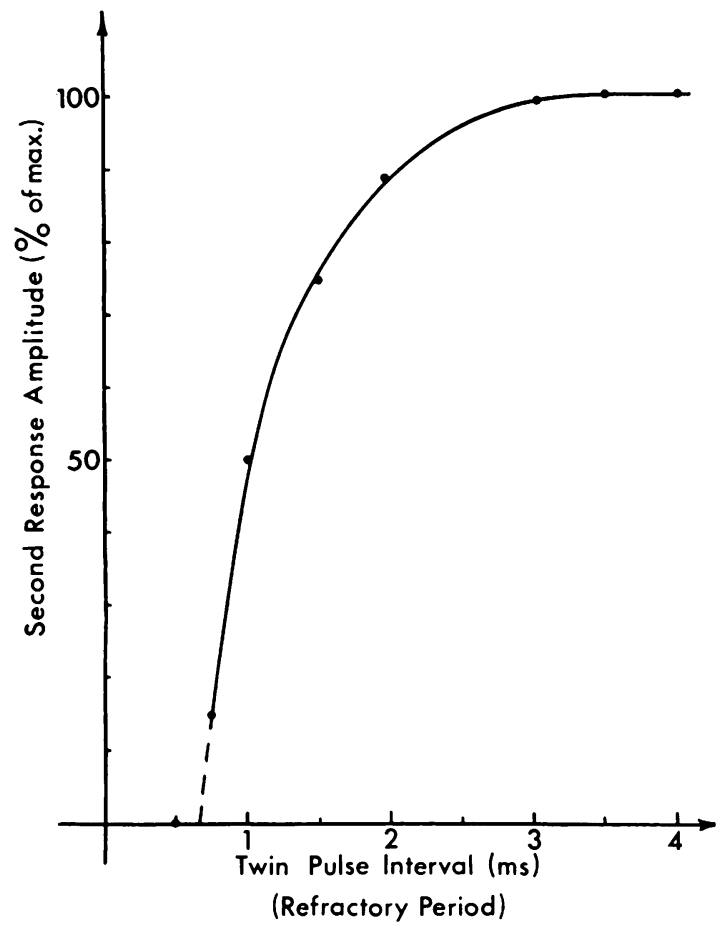

FIG. 4 A graph of the second nerve action potential amplitude (normalized to its maximum value) plotted against the pulse interval (refractory period) taken from a typical $X-Y$ plotter readout as given in Fig. 3.

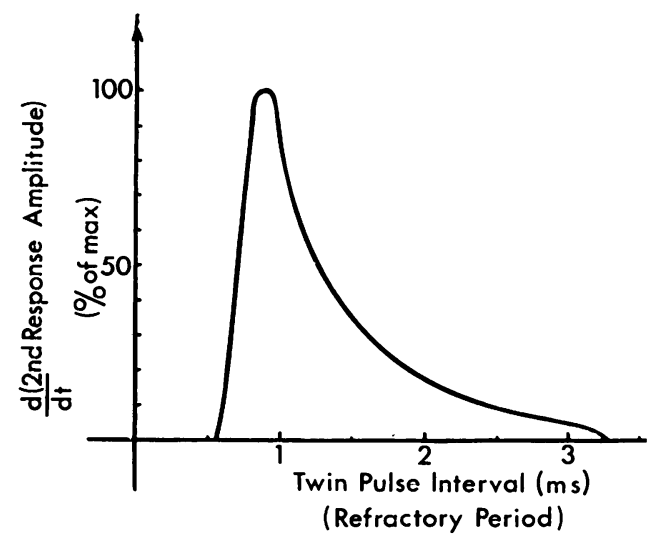

FIG. 5 The distribution of fibre refractory periods, derived by differentiation of the amplitude versus time curve as given in Fig. 4. duct the second of a pair of stimuli was called the 'critical interval of conduction' by Tasaki (1959), who quotes values of $0.68 \pm 0.06 \mathrm{~ms}$ in young subjects compared with $0.72 \pm 0.05 \mathrm{~ms}$ in older subjects. Gilliatt and Willison (1963) reported absolute refractory periods of $0.6-0.7 \mathrm{~ms}$ in four normal subjects. Buchthal and Rosenfalck (1966) measured a value of $0.75 \mathrm{~ms}$ in sensory fibres of the median nerve. More recently, Lowitzsch and Hopf (1972), Lowitzsch et al. (1973), Tackmann and Lehmann (1974), and Hopf et al. (1975) have discussed, in some detail, refractory period measurements.

The absolute refractory period is the time interval during which a nerve fibre is incapable of conducting another impulse after a conditioning stimulus (see Brazier, 1973). In the present work we have used stimuli of an intensity about $10 \%$ greater than that which elicited the maximal amplitude response. Since the nerve is capable of responding to large stimuli during the relative refractory period, it is probable that the present measurements are of relative rather than absolute refractory period. The distinctions between absolute and relative refractory period measurement are well described in the above literature together with the significance of changes in conduction velocity during relative refractory periods.

The present work has shown that the conduction velocity ranges measured elbow to wrist are within the range $6.9-12 \mathrm{~m} / \mathrm{s}$ which is in good agreement with Chaco (1970) and Miglietta (1968). However, using the identical technique over long nerve lengths, wrist to axilla, the velocity ranges are significantly reduced $(P<$ $0.001)$. The variation of conduction velocity ranges with changes in conduction distance can be explained by the omission of refractory period corrections. Refractory period delays are a significant percentage of the conduction times and cannot be neglected when making measurements of the range of conduction velocities.

Blackstock et al. (1972) have made some attempt to correct for refractory period delays by using a $1 \mathrm{~ms}$ correction factor applied only to the slow conducting fibres. If corrections are to be made, assumptions need to be made about the possible variation of refractory period with fibre conduction velocity. On the assumption that $t_{f}$ and $t_{s}$ are the conduction times plus 
refractory period values of the fast and slow conducting fibres respectively, our calculated refractory periods (Table 1) are within the range 0.4-3.8 ms. However, if these values are then used to correct the velocity range, then very small or indeed negative velocity ranges are obtained.

An alternative assumption of constant refractory period could be made but would be inconsistent with our direct refractory period measurements (Figs. 4 and 5) which give a range of $0.65-3.6 \mathrm{~ms}$. The distribution of refractory periods shown in Fig. 5 is markedly skewed with the majority of fibres having short refractory periods. This skewness of distribution was found in all the cases studied. The median values of refractory period give a mean of $1.30 \pm 0.41 \mathrm{~ms}( \pm 2 \mathrm{SD})$. If varying the time interval between the two pulses applied at a single site selectively stimulates fibres of different refractory periods, and assuming a relationship between the conduction rate and refractory period, then changes in dispersion of the second action potential might have been seen. However, no measurable waveform changes were evident, which would be compatible with the assumption of a constant refractory period. The derivation of the range of refractory periods from the graph in Fig. 4 depends upon the assumption that the change in amplitude of the second nerve action potential is due to block of fibres rather than temporal dispersion. Buchthal and Rosenfalck (1966) attempted to measure single nerve action potentials and quote major peak durations of less than $1 \mathrm{~ms}$. Undispersed fibre nerve action potentials have been calculated (A. T. Barker, personal communication) with a major peak duration of about $1 \mathrm{~ms}$. This is likely to be of the same order as the temporal dispersion over a length of nerve in the arm. Although this is not insignificant, it is unlikely that temporal dispersion will make any major contribution to the interpretation of Figs. 4 and 5. The absence of measurable dispersion of the second nerve action potential supports the above assumption.

On the basis of the above findings, a reasonable hypothesis is that for group A fibres the random scatter of refractory period values is far greater than any variation due to a correlation between refractory period and conduction velocity. Therefore a single refractory period value is the more reasonable correction factor to use when calculating velocity ranges from a double pulse technique. The measurements of refractory period distributions suggest that the median value could be taken. Applying this value of refractory period as a correction to the conduction velocity range test gives velocity ranges in the region of $15-20 \mathrm{~m} / \mathrm{s}$ and gives fast conduction velocities which are in good agreement with those found by direct measurement. This conduction velocity range is of the order that might be expected from the considerable spread of fibre diameter population found in peripheral nerves. Notwithstanding this apparent agreement, the measured ranges of refractory period are significant and cannot be ignored when attempting to measure conduction velocity ranges. This is particularly important when the length of nerve under investigation is short and the refractory period a greater proportion of the conduction times. It must be recognized that calculated values of conduction velocity range are a function not only of the spread of fibre conduction velocities but also of refractory periods.

We would like to thank all the normal subjects who made this study possible. The material used in this paper forms part of a M.Med.Sci. thesis to be submitted to the University of Sheffield.

\section{REFERENCES}

Blackstock, E., Rushworth, G., and Gath, D. (1972)' Electrophysiological studies in alcoholism. Journal of Neurology, Neurosurgery, and Psychiatry, 35, 326-334.

Brazier, M. A. B. (1973). The Electrical Activity of the Nervous System. A Textbook for Students. Pitman Medical: Tunbridge Wells, Kent.

Buchthal, F., and Rosenfalck, A. (1966). Evoked action potentials and conduction velocity in human sensory nerves. Brain Research, suppl. 3, p. 122.

Chaco, J. (1970). Conduction velocity of motor nerve fibres in progressive spinal atrophy. Acta Neurologica Scandinavica, 46, 119-122.

Gilliatt, R. W., and Willison, R. G. (1963). The refractory and supernormal periods of the human median nerve. Journal of Neurology, Neurosurgery, and Psychiatry, 26, 136-147.

Hopf, H. C. (1963). Electromyographic study on socalled mononeuritis. Archives of Neurology (Chic.), 9, 307-312. 
Hopf, H. C., Le Quesne, P. M., and Willison, R. G. (1975). Refractory period and lower limiting frequencies of sensory fibres of the hand. In Studies of Neuromuscular Diseases. Proceedings of Int. Symp. Giessen 1973, pp. 258-263. Edited by K. Kunze and J. E. Desmedt. Karger: Basel.

Lowitzsch, K., and Hopf, H. C. (1972). Refraktärperiode und Übermittlung frequenter Reizserien im gemischten peripheren Nerven des Menschen. Journal of the Neurological Sciences, 17, 255-270.

Lowitzsch, K., Hopf, H. C., and Schlegel, H. J. (1973). Conduction of two or more impulses in relation to the fibre spectrum in the mixed human peripheral nerve. In New Developments in Electromyography and Clinical Neurophysiology, vol. 2, pp. 272-278. Edited by J. E. Desmedt. Karger: Basel.

McComas, A. J., Sica, R. E. P., and Currie, S. (1970). Muscular dystrophy: evidence for a neural factor. Nature, 226, 1263-1264.
Miglietta, O. (1966). Nerve motor fiber characteristics in chronic ischemia. Archives of Neurology (Chic.), 14, 448-453.

Miglietta, O. (1968). Motor nerve fibers in amyotrophic lateral sclerosis. American Journal of Physical Medicine, 47, 118-124.

Tackmann, W., and Lehmann, H. J. (1974). Refractory period in human sensory nerve fibres. European Neurology, 12, 277-292.

Tasaki, I. (1959). Conduction of the nerve impulse. In Handbook of Physiology, vol. 1. Edited by J. Field, H. W. Magoun, and V. E. Hall. American Physiological Society: Washington.

Thomas, P. K., Sears, T. A., and Gilliatt, R. W. (1959). The range of conduction velocity in normal motor nerve fibres to the small muscles of the hand and foot. Journal of Neurology, Neurosurgery, and Psychiatry, 22, 175-181. 